\title{
Preparação de mulita a partir do mineral topázio
}

\section{(Preparation of mullite from topaz)}

\author{
R. R. Monteiro ${ }^{1}$, A. C. S. Sabioni ${ }^{2,3}$, G. M. da Costa ${ }^{3}$ \\ ${ }^{1}$ Av. Central 27, ap. 101, Jd. Savoia, Ilhéus, BA 45658-260 \\ ${ }^{2}$ Laboratório de Difusão em Materiais, Departamento de Física \\ ${ }^{3}$ Departamento de Química \\ Universidade Federal de Ouro Preto, Ouro Preto, MG 35400-000 \\ ticlereis@aol.com,sabioni@iceb.ufop.br,magela@iceb.ufop.br
}

\begin{abstract}
Resumo
Mulita é o único composto termodinamicamente estável no sistema binário $\mathrm{Al}_{2} \mathrm{O}_{3}-\mathrm{SiO}_{2}$, na faixa 70,5 a 74,0\% em peso de $\mathrm{Al}_{2} \mathrm{O}_{3}$. Mulita metaestável na faixa de 74 a $83,6 \%$ de $\mathrm{Al}_{2} \mathrm{O}_{3}$, entretanto, pode ser obtida. Devido às suas excelentes propriedades físicas e mecânicas a altas temperaturas, como alto ponto de fusão, baixa expansão térmica, boa resistência à fratura e ao choque térmico, alta resistência à fluência, estabilidade térmica, baixa densidade e baixa constante dielétrica, tem tido um uso cada vez maior em corpos cerâmicos. O mineral mulita, porém, é raro e quase inexistente na natureza. Para atender a um mercado crescente, mulitas sintéticas são produzidas, por meio de misturas de pós de $\mathrm{Al}_{2} \mathrm{O}_{3}$ e $\mathrm{SiO}_{2}$ em escala molecular, usando técnica sol-gel, ou por meio da calcinação de minerais que contenham sílica e alumina em suas estruturas, os chamados alumino-silicatos. Normalmente estes minerais contêm impurezas e, muitas vezes, produzem uma mulita acompanhada de uma fase vítrea. Neste trabalho é feito o estudo alternativo para se obter uma mulita pura e de baixo custo por meio da calcinação do topázio $\mathrm{Al}_{2} \mathrm{SiO}_{4}\left[\mathrm{~F}_{\mathbf{x}}(\mathrm{OH})_{1-\mathrm{x}}\right]_{2}$. Topázio incolor e imperial foram utilizados para a produção de mulita. O topázio incolor não tem valor gemológico nem comercial e é abundante na natureza. Para o topázio imperial foram usados refugos provenientes de sua extração. O rendimento da calcinação foi alto, cerca de $80 \%$, obtido a uma temperatura não muito alta, em torno de $1300^{\circ} \mathrm{C}$, produzindo uma mulita muito pura e sem fase vítrea. A microestrutura da mulita obtida foi do tipo agulhas (whiskers de mulita) e apresentou alta porosidade. Essa microestrutura e porosidade são duas propriedades intrínsecas que estão associadas à decomposição do topázio. Este estudo mostrou que o topázio é uma fonte alternativa para a produção de mulita de baixo custo e de alta qualidade. Palavras-chave: topázio, mulita.
\end{abstract}

\begin{abstract}
Mullite is the unique intermediate compound thermodynamically stable in the binary system $\mathrm{Al}_{2} \mathrm{O}_{3}-\mathrm{SiO}_{2}$, in the weight range from 70.5 to $74.0 \%$ of $\mathrm{Al}_{2} \mathrm{O}_{3}$. However mullite metastable in the range from 74 to $83.6 \%$ of $\mathrm{Al}_{2} \mathrm{O}_{3}$ can be obtained. Due to its excellent physical and mechanical properties at high temperatures such as high melting point, low thermal expansion, good resistance to fracture and to thermal shock, high creep resistance, thermal stability, low density and low dielectric constant, it has become widely used in ceramic bodies. However, the mineral mullite is rare and almost nonexistent in the nature. To better assist to a growing market, synthetic mullites are produced, by mixtures of powders of $\mathrm{Al}_{2} \mathrm{O}_{3}$ and $\mathrm{SiO}_{2}$ in molecular scale, by using sol-gel technique, or by the calcination of minerals that contains silica and alumina in their structures, so called alumino silicates. Usually these minerals contain impurities, and as consequence, they produce mullite accompanied by a glassy phase. In this work showed that the topaz is another alternative to obtaining a pure and cheap mullite through the calcination of the topaz $\mathrm{Al}_{2} \mathrm{SiO}_{4}\left[\mathrm{~F}_{x}(\mathrm{OH})_{1-x}\right]_{2}$. Colorless topaz and imperial topaz were used for the mullite production. The colorless topaz doesn't have commercial or gemological value, it is quite abundant. For the imperial topaz it was used drosses of its extraction. The yield in the calcination was high, around 80\%, and it was obtained at a temperature not very high, around $1300^{\circ} \mathrm{C}$, producing a very pure mullite without glassy phase. The microstructure of the obtained mullite was of needle type (mulitte whiskers) and high porosity. These two features are intrinsic properties that are associated to the topaz decomposition. This study showed that the topaz may be an inexpensive source for obtaining mullite of high quality.
\end{abstract}

Keywords: topaz, mullite.

\section{INTRODUÇÃO}

A mulita, devido às suas excelentes propriedades físicas, tais como resistência à fluência a altas temperaturas, estabilidade química, baixa constante dielétrica e baixo coeficiente de expansão térmica, tem chamado ultimamente a atenção de muitos pesquisadores [1]. Como mineral, a mulita é rara na natureza [2] e s depósitos atuais não são suficientes para abastecer um mercado crescente em função das novas aplicações encontradas. Isso faz com que muitos pesquisadores procurem sintetizá-la, principalmente através de misturas de $\mathrm{Al}_{2} \mathrm{O}_{3}$ e $\mathrm{SiO}_{2}$, ou a partir de minerais que apresentam $\mathrm{Al}_{2} \mathrm{O}_{3} \mathrm{e}$ $\mathrm{SiO}_{2}$ em suas composições. Quase todos esses processos resultam em uma mulita com uma fase vítrea presente. A síntese 
de mulita através de técnicas "sol-gel” tem produzido mulita pura, mas a um custo elevado [3].

Um processo alternativo para a produção de uma mulita de alta pureza é através da calcinação do topázio incolor e imperial. O topázio incolor é um mineral abundante na natureza, sem valor gemológico e sem uma aplicação econômica que viabilize a sua exploração. O topázio imperial possui um alto valor gemólogico, mas cerca de $95 \%$ do mineral extraído é rejeitado. Dos 5\% restantes, quase a metade é descartada durante o processo de lapidação. Este rejeito tem tido pouquíssimas aplicações que justifiquem o seu uso. Portanto, o objetivo principal deste trabalho é investigar as características físico-químicas da mulita obtida a partir do topázio incolor e do topázio imperial.

\section{MATERIAIS E MÉTODOS}

Amostras de topázio incolor de localidades do nordeste de Minas Gerais e topázio imperial da região de Ouro Preto (MG) foram selecionadas com base em seus aspectos macroscópicos. Após fragmentação, o material foi pulverizado durante 80 minutos em um moinho tipo panela-disco. Os resultados das análises químicas de duas amostras típicas estão apresentados na Tabela I.

A análise granulométrica foi efetuada no analisador de tamanho de partículas Sedigraph 5100 Micromeritics. O diâmetro das partículas do topázio incolor variou entre 1,0 e $30 \mu \mathrm{m}$ e do topázio imperial entre 1,0 e $50 \mu \mathrm{m}$.

As fases cristalográficas do topázio e das mulitas obtidas foram identificadas em um difratômetro Shimadzu XRD-6000 equipado com tubo de cobalto e utilizando filtro de ferro. As varreduras foram feitas entre $7^{\circ}$ e $70^{\circ} \mathrm{com}$ velocidade de $0,5 \% \mathrm{~min}$ e utilizando silício como padrão interno. Os parâmetros de redes foram calculados através do programa JADE após subtração do background e da contribuição de $\mathrm{K}_{\alpha{ }_{2}}$.

As análises termogravimétricas (TGA) foram feitas em um aparelho modelo SDT 2960 TA Instruments. A faixa de

Tabela I - Identificação das amostras de topázio tratadas em diferentes temperaturas e tempos de aquecimentos.

[Table I - Identification of the topaz samples treated at various temperatures and periods of time.]

\begin{tabular}{cccc}
\hline Amostra & $\begin{array}{c}\text { Tratamento } \\
\text { térmico }\left({ }^{\circ} \mathrm{C}\right)\end{array}$ & Topázio & Estado \\
\hline $\mathrm{A}$ & $1300 / 3 \mathrm{~h}$ & Incolor & Pastilha \\
\hline $\mathrm{B}$ & $1300 / 3 \mathrm{~h}$ & Incolor & Cristal \\
\hline $\mathrm{C}$ & $1300 / 2 \mathrm{~h}$ & Imperial & Pó \\
\hline $\mathrm{D}$ & $1300 / 1 \mathrm{~h}$ & Incolor & Pó \\
\hline $\mathrm{E}$ & $1300 / 1 \mathrm{~h}$ & Incolor & Cristal \\
\hline $\mathrm{F}$ & $1200 / 10 \mathrm{~min}$ & Incolor & Pastilha \\
\hline $\mathrm{G}$ & $1100 / 1 \mathrm{~h}$ & Incolor & Pó \\
\hline $\mathrm{H}$ & $1100 / 13 \mathrm{~h} 20 \mathrm{~min}$ & Incolor & Pó \\
\hline
\end{tabular}

temperatura variou da ambiente até $1380{ }^{\circ} \mathrm{C}$, usando um fluxo constante de nitrogênio $(100 \mathrm{~mL} / \mathrm{min})$ ou ar estático e uma taxa de aquecimento de $10^{\circ} \mathrm{C} / \mathrm{min}$.

Pastilhas de pó de topázio, umedecidas com álcool etílico ou nujol, foram prensadas uniaxialmente a $31 \mathrm{MPa}$ por $5 \mathrm{~min}$. As pastilhas, o pó de topázio e um cristal de topázio incolor foram tratados em ar estático a temperaturas variando desde à ambiente até $1300{ }^{\circ} \mathrm{C}$. O tempo de tratamento das amostras variou de $1 \mathrm{~h}$ a $14 \mathrm{~h}$, nas temperaturas desejadas. As amostras tratadas foram identificadas conforme a Tabela I.

A microestrutura das amostras de mulita foi observada em um microscópio de varredura eletrônica fabricado pela Jeol JSM 5510.

\section{RESULTADOS}

\section{Transformação do topázio em mulita}

A Fig. 1 mostra o efeito do aquecimento entre $1000{ }^{\circ} \mathrm{C}$ e $1300{ }^{\circ} \mathrm{C}$ durante uma hora na estabilidade do topázio. Observase que a $1100{ }^{\circ} \mathrm{C}$ começam a aparecer os primeiros traços de mulita, enquanto que a $1200{ }^{\circ} \mathrm{C}$ ainda permanecem pequenas quantidades de topázio. A transformação é completa a $1300^{\circ} \mathrm{C}$,

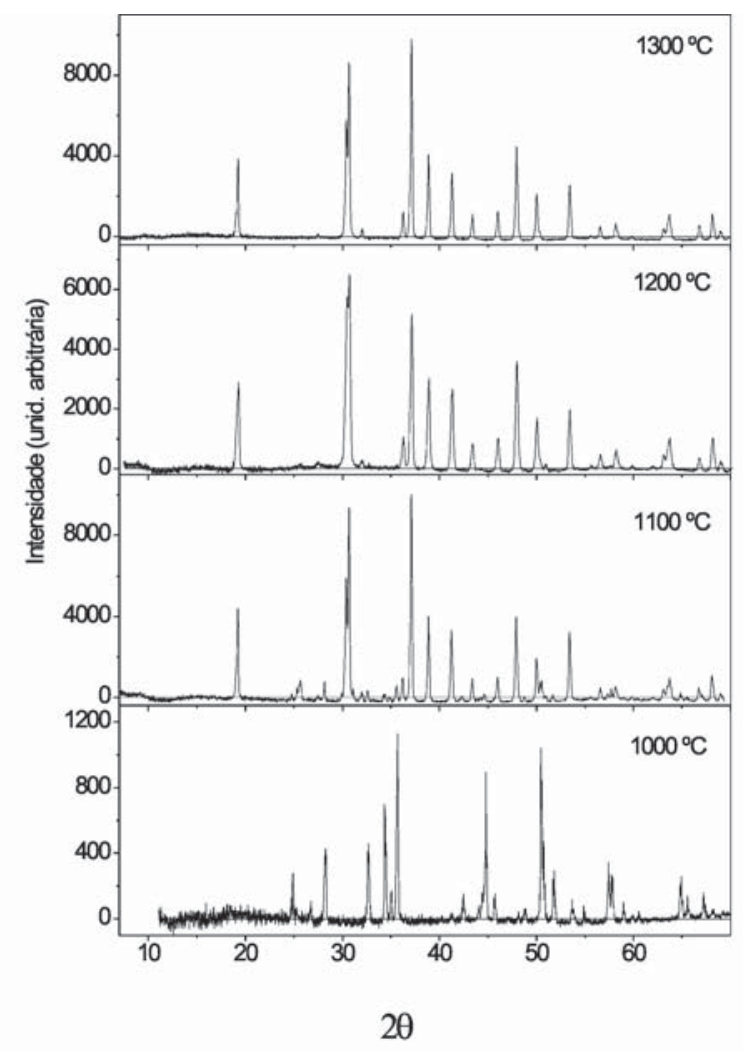

Figure 1: Difratogramas de raios $\mathrm{X}$ de amostras de topázio incolor tratadas em diferentes temperaturas durante $1 \mathrm{~h}$.

[Figure 1: X-ray diffraction patterns of colorless topaz treated at a few selected temperatures during 1 h.] 


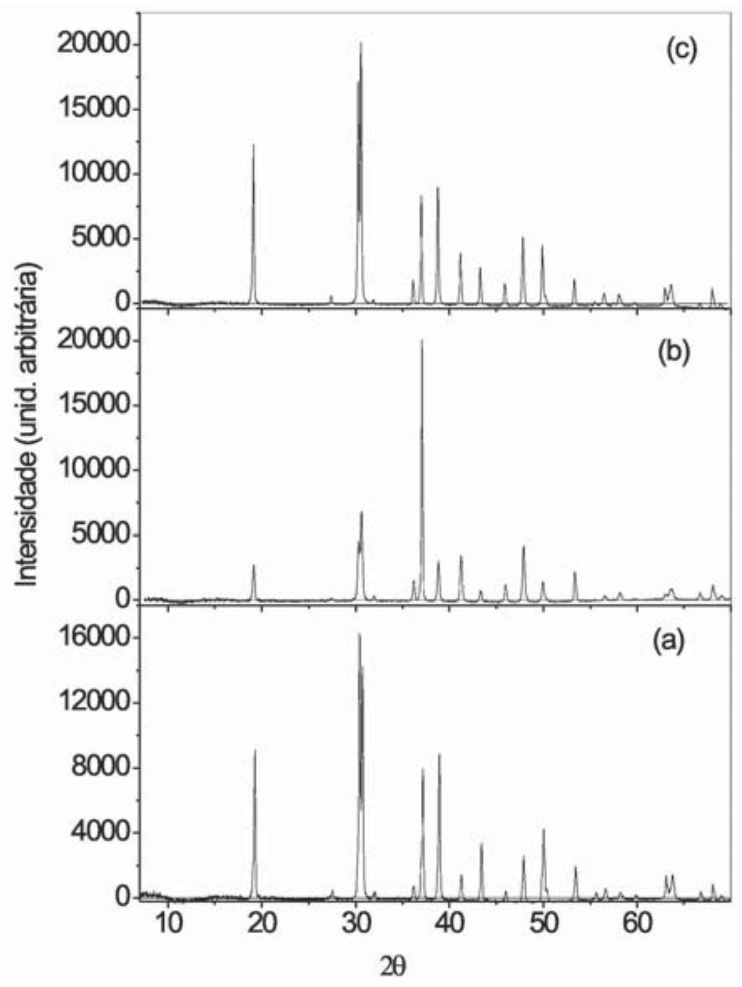

Figura 2: Difratogramas de raios $\mathrm{X}$ de topázio tratado a $1300{ }^{\circ} \mathrm{C}$ durante $1 \mathrm{~h}$ (a), $2 \mathrm{~h}$ (b) e $3 \mathrm{~h} \mathrm{(c).}$

[Figure 2: X-ray diffraction patterns of topaz treated at $1300{ }^{\circ} \mathrm{C}$ during $1 h(a), 2 h(b)$ and $3 h(c)$.

sendo a mulita a única fase observada. A ausência de fase vítrea foi verificada em todas as amostras tratadas a $1300{ }^{\circ} \mathrm{C}$ durante diferentes tempos de aquecimento (Fig. 2).

A amostra E foi caracterizada por porosimetria de mercúrio para a determinação de porosidade e tamanho de poros. Os resultados encontrados (Tabela II) indicam uma porosidade de $15,5 \%$. Os gases liberados durante a decomposição do topázio certamente são os responsáveis pela porosidade aberta observada. Tamanhos de poros na faixa de 0,17 - 0,61 $\mu \mathrm{m}$ ainda não são suficientemente grandes para fornecer permeabilidade à água ou gás, se o propósito para o uso desta mulita porosa é filtro [7]. Porém se esta porosidade é baixa para ser usada como filtro, ela é considerada alta se o propósito for para o uso de corpo cerâmico denso, como por exemplo tijolo refratário. Portanto, a porosidade em mulita obtida a partir do topázio é um aspecto microestrutural intrínseco.

A micrografia da mulita formada (Fig. 3) revelou uma microestrutura tipo agulha, isto é, monocristais na forma de filamentos (whiskers). Suas extremidades têm a forma tetragonal, com duas faces triangulares compartilhando juntas uma das arestas dos triângulos formados [13]. A espessura das agulhas foi calculada como sendo de $1 \mu \mathrm{m}$, e o comprimento inferior a $10 \mu \mathrm{m}$.

A Fig. 4 mostra a microestrutura da mulita referente à amostra "A". A espessura da agulha é próxima de 1,70 $\mu \mathrm{m}$ e o comprimento superior a $50 \mu \mathrm{m}$. Com essas dimensões, é

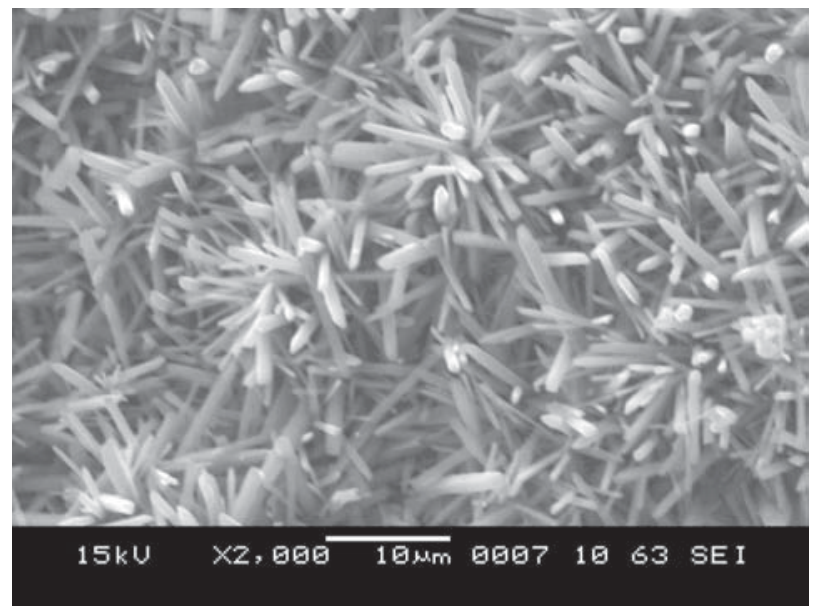

Figura 3: Micrografia MEV de mulita obtida após $1300{ }^{\circ} \mathrm{C}$ durante $1 \mathrm{~h}$ (amostra D).

[Figure 3: SEM micrograph of the mullite obtained after $1300{ }^{\circ} \mathrm{C}$ during 1 h (sample D).]

Tabela II - Porosidade da mulita proveniente da decomposição do topázio incolor tratado a $1300{ }^{\circ} \mathrm{C}$ durante uma hora (amostra E).

[Table II - Porosity of mullite derived from the decomposition of colorless topaz at $1300{ }^{\circ} \mathrm{C}$ during one hour (Sample E).]

\begin{tabular}{ll}
\hline Área total de poros $\left(\mathrm{m}^{2} / \mathrm{g}\right)$ & 3,16 \\
\hline Diâmetro médio (volume) $(\mu \mathrm{m})$ & 0,19 \\
\hline Média do diâmetro $(4 \mathrm{~V} / \mathrm{A})(\mu \mathrm{m})$ & 0,075 \\
\hline Densidade volumétrica $\left(\mathrm{g} / \mathrm{m}^{3}\right)$ & 2,61 \\
\hline Porosidade $(\%)$ & 15,5 \\
\hline
\end{tabular}

encontrada uma ampla aplicação na fabricação de materiais compósitos, isolantes térmicos e outras aplicações envolvendo ambientes de altas temperaturas [13]. O intercrescimento dos cristais de agulhas de mulita concede resistência mecânica e rigidez estrutural ao corpo de mulita porosa [15].

A formação de cristais de mulita na forma de agulhas (whiskers) foi encontrada em todas as amostras de topázio tratadas. Essa propriedade de formar agulhas representa um aspecto microestrutural intrínseco associado com a decomposição do topázio. Comparando as Figs. 3 e 4, podemos observar que o tratamento térmico a $1300{ }^{\circ} \mathrm{C}$, durante, $3 \mathrm{~h}$, é o ideal para garantir as agulhas com dimensões que não ofereçam riscos à saúde, ou seja, com comprimentos superiores a $1 \mu \mathrm{m}$. As análises de difração feitas nessas duas amostras não revelaram nenhuma fase que não fosse mulita.

Muitos métodos têm sido desenvolvidos para produzir mulita com microestrutura tipo agulhas para fabricação de compósitos e muitos deles são caros [13]. Devido ao diâmetro pequeno e à perfeição cristalina, possuem resistência extremamente alta e podem ser empregadas como reforços em compósitos cerâmicos [13].

O pó gerado no manuseio de mulitas "tipo agulhas" pode 

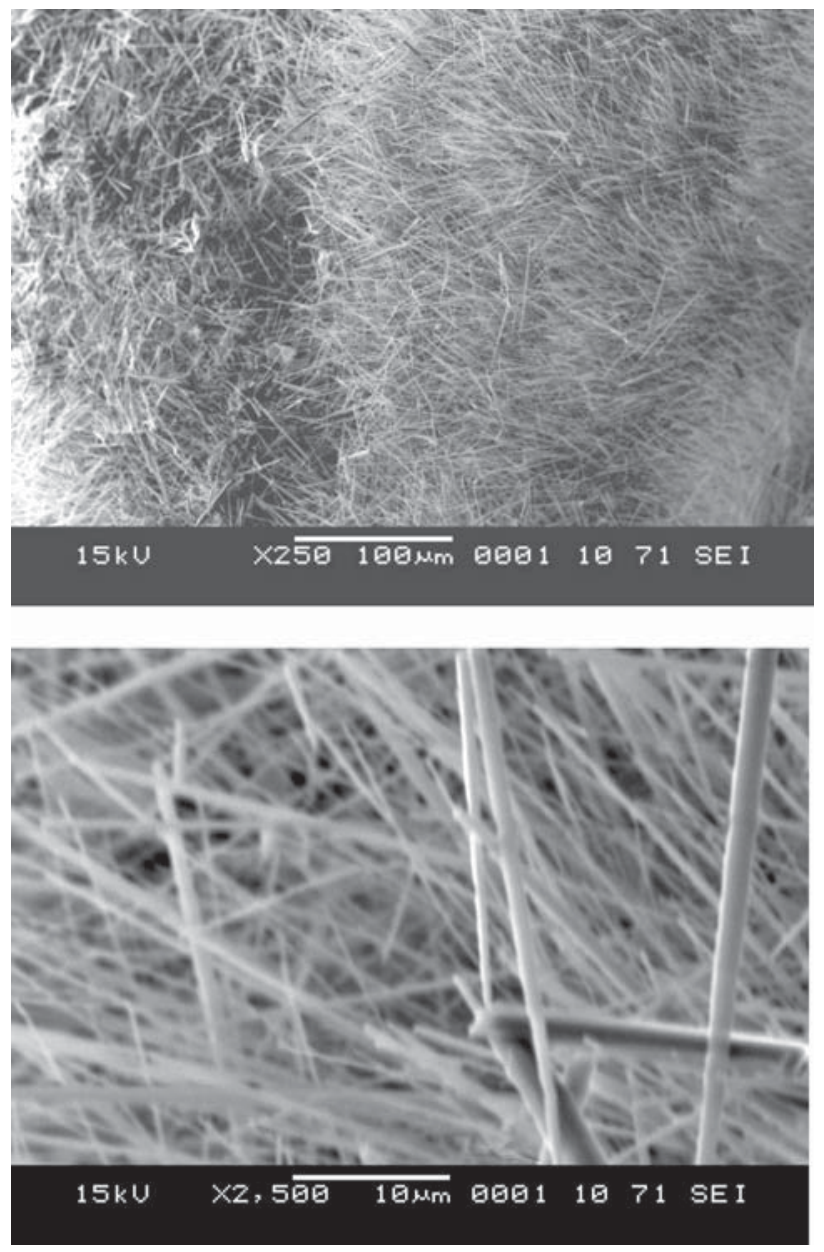

Figura 4: Micrografias MEV de mulita proveniente da decomposição do topázio incolor aquecido a $1300{ }^{\circ} \mathrm{C}$ durante $3 \mathrm{~h}$. Nota-se a formação da microestrutura tipo agulha e o entrelaçamento destas agulhas (amostra A).

[Figure 4: SEM micrographs of the mullite derived from the decomposition of colorless topaz heated at $1300{ }^{\circ} \mathrm{C}$ during $3 \mathrm{~h}$. The formation of microstructure of the needle types is observed as a fiber network (sample A).]

Tabela III - Comparação entre os parâmetros de rede e volume da mulita e do topázio.

[Table III - Comparasion between the cell parameters and volume of mulite and topaz.]

\begin{tabular}{lllll}
\hline Amostra & $\mathrm{a}(\mathrm{nm})$ & $\mathrm{b}(\mathrm{nm})$ & $\mathrm{c}(\mathrm{nm})$ & $\mathrm{V}(\mathrm{nm})^{3}$ \\
\hline Mulita $\left(1300^{\circ} \mathrm{C} / 3 \mathrm{~h}\right)$ & 0,7569 & 0,7686 & 0,2883 & 0,1677 \\
\hline Topázio incolor & 0,6469 & 0,8805 & 0,8387 & 0,3433 \\
\hline
\end{tabular}

ser altamente prejudicial à saúde. Relatórios publicados salientam que cerca de $1 \mu \mathrm{m}$ é justamente o tamanho que leva a pneumoconiose (doença que afeta os pulmões) enquanto que tamanho de "agulhas" maiores ou menores que $1 \mu \mathrm{m}$ provocam menos danos à saúde [14].

\section{DISCUSSÃO}

O mecanismo para a decomposição térmica do topázio ainda não está muito claro e as reações propostas para descrever a transformacao topázio-mulita são as seguintes [4]:

$$
\begin{aligned}
& 6 \mathrm{Al}_{2} \mathrm{SiO}_{4}\left(\mathrm{~F}_{0,75}, \mathrm{OH}_{0,25}\right)_{2} \rightarrow 2\left(3 \mathrm{Al}_{2} \mathrm{O}_{3} \cdot 2 \mathrm{SiO}_{2}\right) \downarrow+ \\
& 2 \mathrm{SiF}_{4} \uparrow+\mathrm{HF} \uparrow+\mathrm{H}_{2} \mathrm{O} \uparrow \\
& 6 \mathrm{Al}_{2} \mathrm{SiO}_{4}\left(\mathrm{~F}_{0,75}, \mathrm{OH}_{0,25}\right)_{2}+54 \mathrm{SiF}_{4}+27 \mathrm{HF}+27 \mathrm{H}_{2} \mathrm{O} \rightarrow \\
& 12 \mathrm{AlOF}+60 \mathrm{SiF}_{4} \uparrow+42 \mathrm{H}_{2} \mathrm{O} \uparrow \rightarrow
\end{aligned}
$$

$2\left(3 \mathrm{Al}_{2} \mathrm{O}_{3} \cdot 2 \mathrm{SiO}_{2}\right) \downarrow+56 \mathrm{SiF}_{4} \uparrow+28 \mathrm{HF} \uparrow+28 \mathrm{H}_{2} \mathrm{O} \uparrow$

A razão Al/Si no topázio é 2 e na mulita é 3. Assim, a remoção de Si do topázio é necessária para a formação de mulita, que ocorre conforme as reações acima, produzindo gás $\mathrm{SiF}_{4}$.

A decomposição química do topázio resulta, portanto, na perda de flúor, hidrogênio (ou água) e silício [4]. Até $1000{ }^{\circ} \mathrm{C}$ as concentrações de $\mathrm{F}$ e $\mathrm{H}$ nas amostras tratadas são constantes e iguais às concentrações nas amostras não tratadas. Acima de $1000{ }^{\circ} \mathrm{C}$ existe uma perda constante de ambos os elementos, mas a perda de hidrogênio torna-se mais rápida a $1100{ }^{\circ} \mathrm{C}$, enquanto a perda de flúor intensifica-se quando a temperatura atinge $1200{ }^{\circ} \mathrm{C}$ [5]. Esta perda de massa está de acordo com várias análises termogravimétricas (TGA), feitas tanto para o topázio incolor como imperial (Fig. 5). Uma perda de massa de aproximadamente $20 \%$ foi observada nestas análises.

A Tabela III mostra os parâmetros de rede e o volume da célula unitária de uma amostra de mulita tratada a $1300{ }^{\circ} \mathrm{C} / 3$ h, e de uma amostra de topázio incolor.

Os parâmetros de rede de várias mulitas tratadas em temperaturas e tempos diferentes, bem como os volumes das células unitárias e as densidades dessas amostras, estão apresentados na Tabela IV. Os parâmetros estão de acordo com a literatura, mantendo sempre a relação $a<b$. Os volumes e as densidades mantêm-se quase constantes, com variações pouco sensíveis, mas obedecendo a uma relação linear com o parâmetro "a" [8-10].

Uma relação empírica para obter uma estimativa da composição da mulita se baseia na constante "a" do reticulado. Conhecendo o valor do parâmetro "a", em Angstrom (Å), pode-se calcular a percentagem molar " $m$ " de $\mathrm{Al}_{2} \mathrm{O}_{3}$ na mulita por meio da seguinte equação $[11,12]$ :

$$
m \%=144,5 \cdot a-1029,5
$$

A fórmula química da mulita foi deduzida como sendo $\mathrm{Al}_{4+2 \mathrm{x}} \mathrm{Si}_{2-2 \mathrm{x}} \mathrm{O}_{10-\mathrm{x}}$ onde " $\mathrm{x}$ " representa o número de lacunas de 


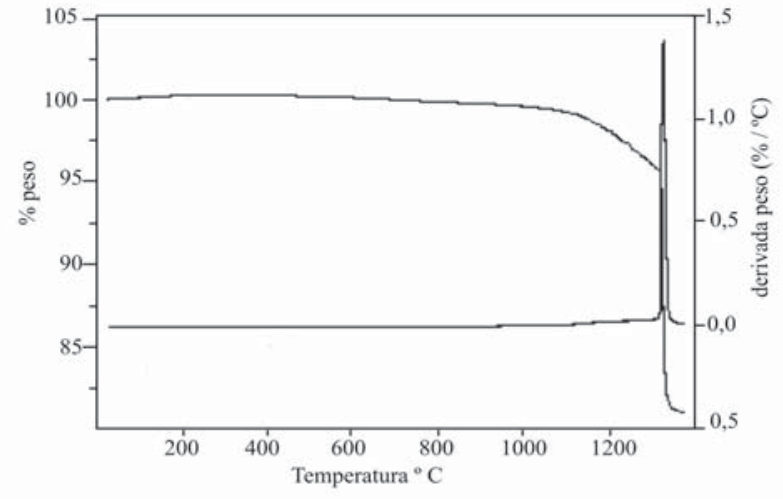

Figura 5: Curva termogravimétrica do topázio incolor. [Figure 5: Thermogravimetric curve of colorless topaz.]

Tabela V - Valores da variação composicional ( $x$ in $\left.\mathrm{Al}_{4+2 \mathrm{x}} \mathrm{Si}_{2-2 \mathrm{x}} \mathrm{O}_{10-\mathrm{x}}\right)$, concentração molar de $\mathrm{Al}_{2} \mathrm{O}_{3}(\mathrm{~m}, \%)$, percentagem em peso de $\mathrm{Al}_{2} \mathrm{O}_{3}$ e relação $\mathrm{Al}_{2} \mathrm{O}_{3}: \mathrm{SiO}_{2}$ das amostras de mulita.

[Table V - Values of compositional variation ( $x \mathrm{em}$ $\left.\mathrm{Al}_{4+2 \mathrm{x}} \mathrm{Si}_{2-2 \mathrm{x}} \mathrm{O}_{10-x}\right), \mathrm{Al}_{2} \mathrm{O}_{3}$ molar concentration ( $\mathrm{m}$, \%), weight percentage of $\mathrm{Al}_{2} \mathrm{O}_{3}$ and ratio $\mathrm{Al}_{2} \mathrm{O}_{3}: \mathrm{SiO}_{2}$ in the mullite samples.]

\begin{tabular}{ccccc}
\hline Amostra & $\mathrm{x}$ & $\mathrm{m}(\%)$ & $\mathrm{Al}_{2} \mathrm{O}_{3}(\%)$ & $\mathrm{Al}_{2} \mathrm{O}_{3}: \mathrm{SiO}_{2}$ \\
\hline $\mathrm{A}$ & 0,347 & 64,27 & 75,32 & $1,80: 1,0$ \\
\hline $\mathrm{B}$ & 0,33 & 63,73 & 74,68 & $1,74: 1,0$ \\
\hline $\mathrm{C}$ & 0,31 & 62,57 & 73,96 & $1,67: 1,0$ \\
\hline $\mathrm{D}$ & 0,35 & 64,41 & 75,41 & $1,81: 1,0$ \\
\hline $\mathrm{F}$ & 0,36 & 64,84 & 75,78 & $1,84: 1,0$ \\
\hline $\mathrm{G}$ & 0,33 & 63,52 & 74,68 & $1,74: 1,0$ \\
\hline $\mathrm{H}$ & 0,36 & 64,86 & 75,78 & $1,84: 1,0$ \\
\hline
\end{tabular}

oxigênio por célula unitária [8]. O valor de "x" pode ser determinado através da concentração molar de $\mathrm{Al}_{2} \mathrm{O}_{3}$, conforme a equação abaixo [11]:

$$
x=10-6\left[\frac{m+200}{m+100}\right]
$$

É importante conhecer o valor do parâmetro "a", pois ele permite concluir se a mulita é estequiométrica, isto é, 3:2 ou $2: 1$. Uma mulita com $\mathrm{m}=60,0 \% \mathrm{e} \mathrm{x}=0,25$ corresponde à mulita $3: 2$ enquanto que para $\mathrm{m}=66,7 \%$ e $\mathrm{x}=0,40$, tem-se a mulita 2:1. Neste espaço composicional entre as mulitas 3:2 e $2: 1$, "m" pode variar de $60,0 \%$ até $66,7 \%$ e, "x" pode variar de 0,25 até $0,40[3,8]$.

Para as mulitas obtidas neste trabalho, as concentrações molares "m" de $\mathrm{Al}_{2} \mathrm{O}_{3}$ foram calculadas através da equação 1, utilizando-se o valor do parâmetro "a" de cada amostra. Os valores da variável composicional " $\mathrm{x}$ " foram obtidos através da equação 2. Os resultados encontrados estão apresentados na Tabela $\mathrm{V}$, de onde se conclui que o topázio forma uma mulita próxima à relação $2: 1$, rica em alumina, com concentração molar de $\mathrm{Al}_{2} \mathrm{O}_{3}$ próxima de $64 \%$ e porcentagens em peso de $\mathrm{Al}_{2} \mathrm{O}_{3}$ próximas de $75 \%$.

\section{CONCLUSÕES}

A decomposição térmica de topázio incolor ou imperial a $1300{ }^{\circ} \mathrm{C}$ durante $3 \mathrm{~h}$ produz uma mulita porosa, com microestrutura tipo agulha e sem fase vítrea. As agulhas apresentam comprimentos superiores a $50 \mu \mathrm{m}$ e diâmetro em torno de $1,70 \mu \mathrm{m}$. Nesta faixa de dimensão, a mulita além de não ser prejudicial à saúde encontra uma ampla aplicação na fabricação de materiais compósitos, isolantes térmicos, catalisadores, filtros para gases a temperaturas elevadas e muitas outras envolvendo ambientes de altas temperaturas.

A análise de porosidade mostrou um corpo cerâmico com porosidade de $15,5 \%$ e com diâmetro médio dos poros de $0,19 \mu \mathrm{m}$.

Tabela IV - Parâmetros de rede, volumes e densidades das mulitas provenientes da decomposição do topázio. [Table IV - Cell parameters, volumes and densities of mulite originating from the decomposition of topaz.]

\begin{tabular}{cccccc}
\hline Amostra & $\mathrm{a}(\AA)$ & $\mathrm{b}(\AA)$ & $\mathrm{c}(\AA)$ & $\mathrm{V}(\AA)$ & $\mathrm{d}\left(\mathrm{g} / \mathrm{cm}^{3}\right)$ \\
\hline $\mathrm{A}$ & $7,569(3)$ & $7,686(3)$ & $2,883(1)$ & 167,8 & 3,16 \\
\hline $\mathrm{B}$ & $7,565(1)$ & $7,688(1)$ & $2,885(1)$ & 167,9 & 3,16 \\
\hline $\mathrm{C}$ & $7,557(2)$ & $7,683(2)$ & $2,883(1)$ & 167,4 & 3,17 \\
\hline $\mathrm{D}$ & $7,570(1)$ & $7,683(1)$ & $2,884(1)$ & 167,8 & 3,16 \\
\hline $\mathrm{F}$ & $7,573(1)$ & $7,687(1)$ & $2,886(1)$ & 168,1 & 3,16 \\
\hline $\mathrm{G}$ & $7,564(3)$ & $7,686(3)$ & $2,889(1)$ & 168,0 & 3,16 \\
\hline $\mathrm{H}$ & $7,573(2)$ & $7,687(2)$ & $2,886(1)$ & 168,0 & 3,16 \\
\hline
\end{tabular}


A produção de mulita através da decomposição do topázio é viável e apresenta baixo custo de produção associado à obtenção de uma mulita de alta qualidade, entretanto cuidados especiais devem ser tomados devido às liberações de gases como $\mathrm{SiF}_{4}$ e $\mathrm{HF}$.

\section{AGRADECIMENTOS}

Este trabalho foi parcialmente financiado por CNPq, Fapemig e Fundação Gorceix.

\section{REFERËNCIAS}

[1] S. Satoshi, C. Contreras, H. Juarez, A. Aguilera, J. Serrato, Int. J. Inorg. Mater. 3, 7 (2001) 625.

[2] W. A. Deer, R. A. Howie, J. Zussman (1982). Rock-Forming Minerals, $2^{\text {nd }}$ Ed., Longman Group Limited, London and New York (1982) p. 719-811.

[3] D. G. Goski, W. F. Caley, Canadian Metallurgical Quartely 38, 2 (1999) 119.
[4] R. A. Day, E. R. Vance, D. J. Cassidy, J. Mater. Res. 10 (1995) 2963.

[5] A. C. S. Sabioni, G. M. Costa, J. M. Dereppe, C. Moreaux, C. M. Ferreira, J. Gemmology 28, 5 (2003) 283.

[6] W. A. Deer , R. A. Howie, J. Zussman, An Introduction to the Rock Forming Minerals, $12^{\text {th }}$ Ed., Longman Group Limited (1980) p. 35-48.

[7] Y. F. Liu, X. Q. Liu, H. Wei, G. Y. Meng, Ceram. Int. 27(2001) 1.

[8] W. E. Cameron, American Mineralogist 62 (1977) 747.

[9] W. E. Cameron, Am. Ceram. Soc. Bull. 56,11 (1977)1003

[10] S. Aramaki, R. Roy, J. Am. Ceram. Soc. 45 (1962) 229.

[11] W. H. Taylor, Z. Krist. 689 (1928) 503.

[12] R. X. Fischer, H. Schneider, D. Vol, J. Eur. Ceram. Soc. 16 (1996) 109.

[13] P. Peng, C. Sorrel, Mater. Lett. 58,7-8 (2004)1288.

[14] A. R. Bunsell, M. H. Berger, J. Eur. Ceram. Soc. 20 (2000) 2249.

[15] K. Okada, N. Otsuka, J. Mater. Sci., Lett. 8 (1989) 1052. (Rec. 27/04/04, Ac. 15/10/04) 